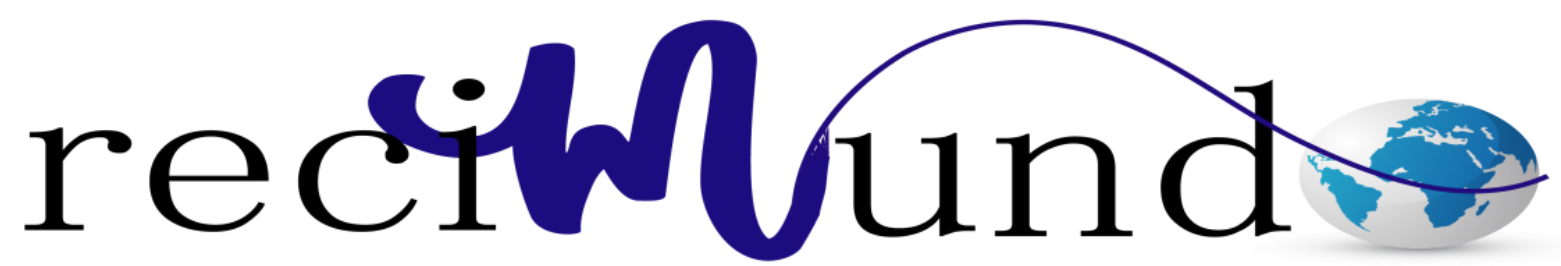

Revista Científica Mundo de la Investigación y el Conocimiento

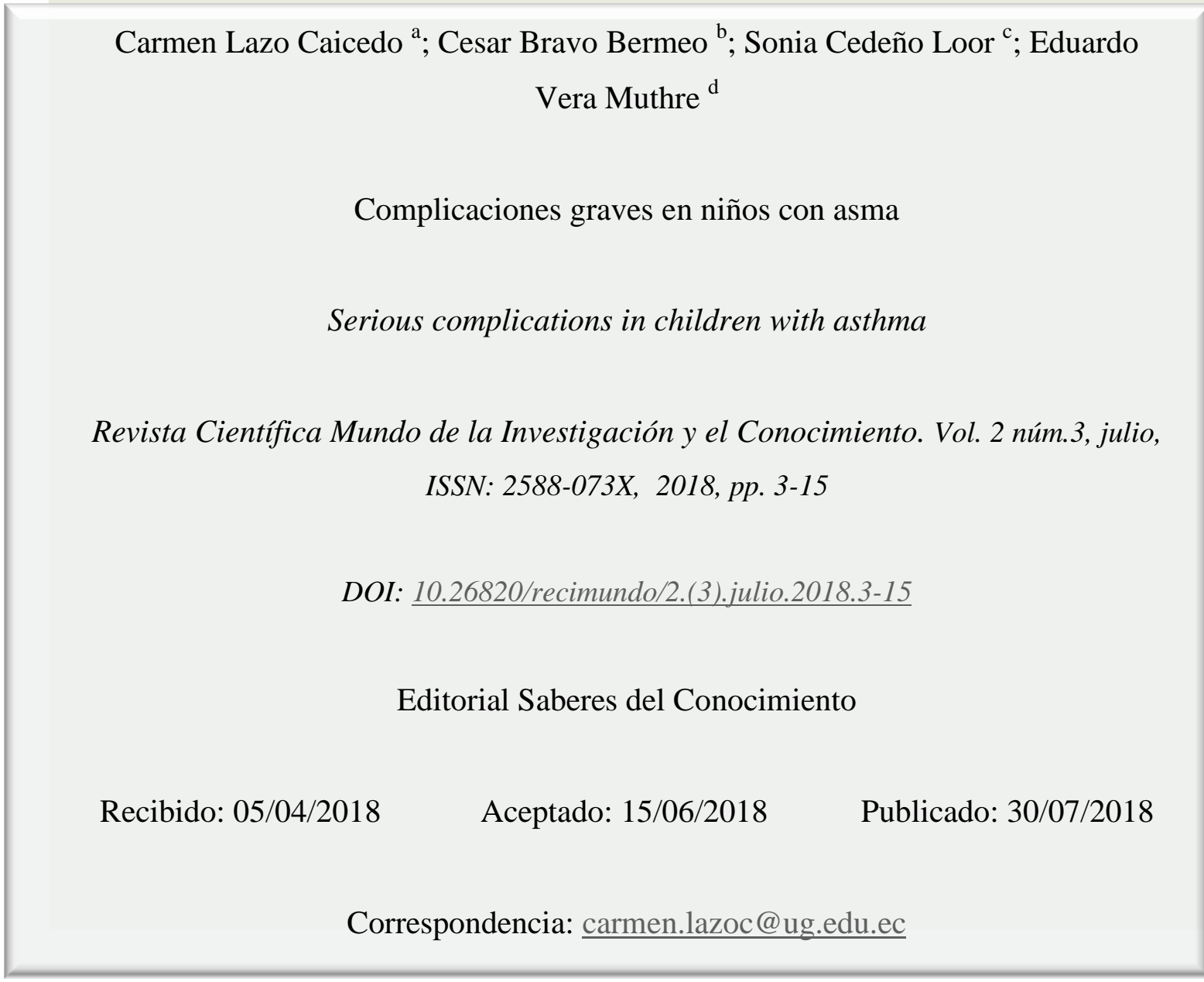

a. Docente Carrera de Medicina. Universidad de Guayaquil; carmen.lazoc@ug.edu.ec

b. Docente Carrera de Medicina. Universidad de Guayaquil; cesar.bravob@ug.edu.ec

c. Docente Carrera de Medicina. Universidad de Guayaquil; leylla.cedenol@ug.edu.ec

d. Docente Carrera de Medicina. Universidad de Guayaquil; eduardo.veram@ug.edu.ec 


\section{Complicaciones graves en niños con asma}

Vol. 2, núm. 3., (2018)

Carmen Lazo Caicedo; Cesar Bravo Bermeo; Sonia Cedeño Loor; Eduardo Vera Muthre

\section{RESUMEN}

Introducción. La morbilidad y mortalidad por asma guardan relación con su gravedad, índices que disminuirían con acciones oportunas y eficaces de las exacerbaciones. Objetivos. Establecer la prevalencia de las complicaciones graves, la presencia de elementos familiares y ambientales, también las dificultades de atención que las determinan. Métodos. Estudio cuantitativo, exploratorio, descriptivo, retrospectivo, no experimental, observacional y documental de las historias clínicas de los pacientes con asma que presentaron complicaciones graves en el Hospital Universitario de la ciudad de Guayaquil, año 2016. Resultados. De un total de 783 preescolares asmáticos, 208 presentaron complicaciones graves (26,5\%). Las complicaciones más frecuentes fueron estatus asmáticos e insuficiencia respiratoria (73), además, alcalosis respiratoria (33), acidosis respiratoria (22) y neumotórax (12). Conclusiones. La elevada frecuencia de complicaciones se da por las condiciones depauperadas de vida, la falta de seguimiento en atención primaria y la inefectiva atención hospitalaria.

Palabras claves: Asma bronquial, Niños, Complicaciones. 


\title{
Complicaciones graves en niños con asma
}

Vol. 2, núm. 3., (2018)

Carmen Lazo Caicedo; Cesar Bravo Bermeo; Sonia Cedeño Loor; Eduardo Vera Muthre

\begin{abstract}
Introducción. Morbidity and mortality due to asthma are related to their severity, rates that would decrease wth a timely and effective actions of exacerbations. Objectives. To establish the prevalence of serious complications, the presence of familiar and enviromenrtal elements and too the attendance difficulties that determine them. Methods. A quantitative, exploratory, descriptive, retrospective, non experimental, observational and documentary study of clinical records of patients with asthma who presented severe complications at the University Hospital of the city of Guayaquil, in 2016 Results. Of a total of 783 asthmatic preschoolers, 208 had severe complications $(26,5 \%)$. The most frecuent complications were asthmatic status and respiratory failure (73), respiratory alcalosis (33), respiratory acidosis (22) and pneumothorax (12). Conclusions. The high frecuency of serious complications is due to poor living conditions, lack of follow-up in primary care and ineffective hospital care.
\end{abstract}

Keywords: Bronchial asthma, Children, Complications. 


\section{Complicaciones graves en niños con asma}

Vol. 2, núm. 3., (2018)

Carmen Lazo Caicedo; Cesar Bravo Bermeo; Sonia Cedeño Loor; Eduardo Vera Muthre

\section{Introducción.}

El asma bronquial tiene un espectro clínico amplio variando de enfermedad leve, intermitente a aquella que es severa, persistente y difícil de tratar, la que en algunas circunstancias puede también ser fatal ${ }^{1}$. Asma padecen alrededor de 235 millones personas del planeta con un porcentaje entre 10-20\% de la población infantil y adolescente (OMS, mayo, 2011). La morbilidad y mortalidad es mayor en los países de ingresos medios y bajos. Las consecuencias del asma en los niños varían en grado, desde no poder asistir a la escuela, el miedo a las crisis y a los ingresos hospitalarios hasta complicaciones graves derivadas de alteraciones de la mecánica respiratoria, desequilibrio acido-base, alteraciones electrolíticas, uso de medicamentos e infecciones agregadas

Según las estimaciones más recientes de la OMS, publicadas en diciembre de 2016, en 2015 hubo 383.000 muertes por asma La muerte por asma, aunque poco común (1 en 2000 asmáticos), se ha incrementado en las últimas décadas con más de 5000 muertes reportadas en USA y 100.000 muertes anualmente a través del mundo ${ }^{2,3}$. Los pacientes con mayor riesgo de ataque fatal de asma son aquellos con enfermedad severa. Las muertes por asma en su mayoría son prevenibles

El asma es una enfermedad inflamatoria crónica, la más frecuente en los niños, cuyas formas severas tienen efecto importante sobre las cifras de morbilidad y mortalidad infantil ${ }^{4}$. El asma severa, aunque difícil de definir incluye todos los casos de difícil tratamiento, incluyendo la administración sistemática de cortocosteroides ${ }^{5}$. Complicaciones de asma: El estatus asmático es la exacerbación asmática severa que no responde al tratamiento médico usual, neumotórax eventualmente asociado con enfisema pulmonar intersticial, neumomediastino, enfisema 


\section{Complicaciones graves en niños con asma}

Vol. 2, núm. 3., (2018)

Carmen Lazo Caicedo; Cesar Bravo Bermeo; Sonia Cedeño Loor; Eduardo Vera Muthre

subcutáneo, neumopericardio, neumoretroperitoneo, fistula traqueo-esofágica, neumonía, sinusitis, toxicidad por teofilina, trastornos electrolíticos, acidosis láctica e hiperglicemia. ${ }^{6,78.9}$. No son raras los taponamientos con moco y atelectasia que responden usualmente al tratamiento.

El estrechamiento de las vías aéreas causa desfase ventilación-perfusión, hiperinflación pulmonar e incremento del trabajo respiratorio que puede conducir a fatiga muscular ventilatoria y falla respiratoria amenazante para la vida ${ }^{10}$.

Por no disponer de estudios previos que con registros numéricos demuestren la magnitud del problema: asma y complicaciones graves, se realizó esta investigación que tuvo como objetivo establecer la frecuencia de las complicaciones graves que presentan los preescolares con asma, ingresados en el Hospital Universitario de la ciudad de Guayaquil en el año 2016, mediante observación directa y recolección de datos de las historias clínicas. Además, identificar y cuantificar variables caracterizadas familiares y ambientales que configuran el diagnóstico de asma $^{11}$.

\section{Objetivos.}

Establecer de forma cuantitativa las complicaciones graves de asma en preescolares que habitan en un sector pobre de la ciudad de Guayaquil, atendidos en la emergencia del Hospital Universitario, año 2016. Así como identificar el nivel de participación de condiciones ambientales y familiares relacionadas con asma. Tiene como propósito resaltar la magnitud del problema y señalar las fallas y dificultades de atención de esta entidad para a partir de su conocimiento elaborar modelos de atención que prevengan el progreso de asma controlable en 


\section{Complicaciones graves en niños con asma}

Vol. 2, núm. 3., (2018)

Carmen Lazo Caicedo; Cesar Bravo Bermeo; Sonia Cedeño Loor; Eduardo Vera Muthre

nivel de atención primaria a asma grave de difícil control en unidades de tercer nivel responsable de los elevados indicadores de morbi-mortalidad.

\section{Métodos.}

Se trata de un estudio observacional y documental, descriptivo, retrospectivo, no experimental, transversal, de abordaje cuantitativo en el que se revisan y analizan historias clínicas y se observan directamente niños de etapa preescolar con asma atendidos en el Hospital Universitario de la Universidad de Guayaquil, año 2016. Los niños incluidos en el estudio pertenecieron a edades comprendidas entre 3 y 5 años, asistían a las unidades de atención primaria durante las exacerbaciones y cuyos familiares aceptaron voluntariamente responder a un determinado número de preguntas relacionadas con la enfermedad.

Fueron atendidos en la emergencia del hospital y se los diagnostico como asma severa por ajustarse a la definición del término: enfermedad resistente al tratamiento médico habitual de todos los grupos de edad. Con las variables consideradas factores de riesgo se elaboró el siguiente cuestionario: 1) contacto domiciliario con perros y/o gatos, 2) antecedentes familiares de asma, 3) tabaquismo familiar, 4) hacinamiento 5) contaminación ambiental. La observación de los pacientes se hizo en las guardias de la emergencia y los datos se obtuvieron del departamento de estadística del mencionado hospital. Universo: 783 pacientes con asma de diferente estadio de presentación del cuadro de acuerdo a la clasificación actual de asma: intermitente leve, persistente leve, moderada y grave. Muestra: 208 con asma severa o grave. Se excluyeron los pacientes de asma que no correspondían al grupo etario y cuyos familiares no aceptaron responder al cuestionario así como también los pacientes que no cumplían el enunciado de asma severa. 


\section{Complicaciones graves en niños con asma}

Vol. 2, núm. 3., (2018)

Carmen Lazo Caicedo; Cesar Bravo Bermeo; Sonia Cedeño Loor; Eduardo Vera Muthre

\section{Resultados.}

El registro de los 208 preescolares con complicaciones graves de asma bronquial atendidos en el Hospital Universitario, el año 2016, revisados y analizados mostro las conclusiones que a continuación se detallan:

1. El asma en los niños continúa con cifras de elevada prevalencia, de manera similar a lo que ocurre tanto en países industrializados como en vías de desarrollo, confirmatorias de que es la enfermedad crónica no transmisible más frecuente en la niñez.

2. Es muy elevado el número de complicaciones graves del asma observadas en relación con el número total de niños asmáticos atendidos en el periodo analizado. 208/783, $(26,5 \%)$

3. Las complicaciones graves más frecuentes se dan a la edad de 5 años (42\%); 4 años, (35\%); 3 años, (23\%). Tabla 1.

4. Destacan en los preescolares analizados el antecedente de asma familiar, exposición a alérgenos de animales: tenencia de gatos y perros, 33\%; tabaquismo en familia, 29\%; antecedente de asma, 18\%; hacinamiento, 14\%; residencia cercana a sitios de contaminación ambiental, 19\%; Tabla 2.

5. Las complicaciones graves más frecuentes fueron : estatus asmático, 35\%; insuficiencia respiratoria grave, 33\%; acidosis respiratoria, 16\%; alcalosis respiratoria, 11\%; neumotórax, 6\%. Tabla 3 . 


\section{Complicaciones graves en niños con asma}

Vol. 2, núm. 3., (2018)

Carmen Lazo Caicedo; Cesar Bravo Bermeo; Sonia Cedeño Loor; Eduardo Vera Muthre

Tabla 1. Edades y frecuencia de complicaciones de asma grave

\begin{tabular}{|l|c|c|}
\hline \multicolumn{1}{|c|}{ PREVALENCIA } & FRECUENCIA & PORCENTAJE \\
\hline 3 años & 48 & $23 \%$ \\
\hline 4 años & 73 & $35 \%$ \\
\hline 5 años & 87 & $42 \%$ \\
\hline Total & 208 & $100 \%$ \\
\hline
\end{tabular}

Tabla 2. Factores de riesgo de asma

\begin{tabular}{|l|c|c|}
\hline \multicolumn{1}{|c|}{ FACTORES DE RIESGO } & FRECUENCIA & PORCENTAJE \\
\hline Tenencia de gatos y perros & 69 & $33 \%$ \\
\hline Tabaquismo en familia & 54 & $29 \%$ \\
\hline Antecedentes de asma & 38 & $18 \%$ \\
\hline Hacinamiento & 29 & $14 \%$ \\
\hline Contaminación de aire & 18 & $19 \%$ \\
\hline
\end{tabular}

Tabla 3. Complicaciones graves de asma

\begin{tabular}{|l|c|c|}
\hline \multicolumn{1}{|c|}{ COMPLICACIONES GRAVES } & FRECUENCIA & PORCENTAJE \\
\hline Estado asmático & 73 & $35 \%$ \\
\hline Insuficiencia espiratoria & 68 & $33 \%$ \\
\hline Alcalosis respiratoria & 33 & $16 \%$ \\
\hline Acidosis respiratoria & 22 & $\mathbf{1 1 \%}$ \\
\hline Neumotórax & 12 & $\mathbf{6 \%}$ \\
\hline Total & 208 & $\mathbf{1 0 0 \%}$ \\
\hline
\end{tabular}

\section{Discusión.}

La morbilidad y mortalidad por asma es un problema de salud pública en muchos países, a pesar de los avances en el conocimiento de esta enfermedad y su tratamiento. Lo que la literatura médica más confiable reporta sobre las complicaciones graves del asma bronquial en niños es la que se elabora en países desarrollados en base a experiencias en hospitales dotados de los recursos técnicos y humanos que permiten afrontar todas las instancias del asma que no 


\section{Complicaciones graves en niños con asma}

Vol. 2, núm. 3., (2018)

Carmen Lazo Caicedo; Cesar Bravo Bermeo; Sonia Cedeño Loor; Eduardo Vera Muthre

responde al tratamiento convencional y requiere de actitudes agresivas tanto farmacológicas como de asistencia ventilatoria en departamentos bien estructurados de cuidados críticos y servicios pulmonares.

En el caso del Hospital Universitario de Guayaquil, la situación es distinta, con muchas limitaciones en lo técnico y en lo humano. Los pacientes con asma severa, en el periodo observado, 2016, en mayoría fueron trasladados a hospitales de niños, por lo que, los registros estadísticos señalan los diagnósticos observables al ingreso y en muy pocos casos la evolución y destino final de los pacientes. Con estas limitaciones lo único que se puede extraer, convertido en objetivo de estudio, es la cuantificación de las complicaciones graves en el grupo de edad escogido y de variables caracterizadas relacionadas con asma bronquial.

Con este antecedente se puede señalar una revisión de asma severa realizada en la unidad de cuidados intensivos pediátricos de un hospital español en el que se trataron 77 veces 64 pacientes en edades de 0,5 a 13,9 años de edad, los menores de 5 años representaban el 50\%, este dato concuerda con los resultados del grupo etario (preescolar) registrado en esta revisión local, que identifica la edad de los niños en la que se observan las complicaciones graves de esta entidad.

La revisión casuística demuestra que el neumotórax y el neumomediastino son complicaciones frecuentes en pacientes con asma severa y la bibliografía señala que estas se presentan como resultado de la ventilación mecánica, en apoyo de esta realidad un estudio retrospectivo de 79 niños de una población de 2142 asmáticos hospitalizados admitidos durante un periodo de 32 meses a la UCI para el tratamiento de estatus asmático severo. 19 requirieron ventilación mecánica debido al incremento de la fatiga y empeoramiento del broncoespasmo. De 


\section{Complicaciones graves en niños con asma}

Vol. 2, núm. 3., (2018)

Carmen Lazo Caicedo; Cesar Bravo Bermeo; Sonia Cedeño Loor; Eduardo Vera Muthre

ellos, 2 desarrollaron neumotórax que se resolvieron sin incidentes; 5 no desarrollaron neumotórax pero antes de la intubación tuvieron fuga de aire al mediastino y subcutánea. En el registro del hospital se anota la presencia de casos de neumotórax sin conocer su evolución posterior.

De las complicaciones graves observadas en esta revisión analítica de asma en niños, el

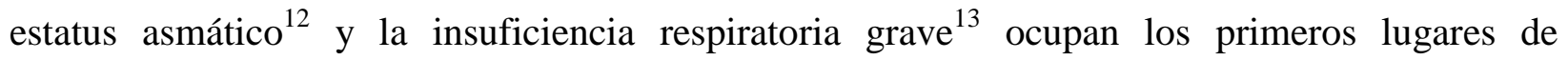
frecuencia, información que coincide con los estudios disponibles. El estatus asmático es la forma extrema de exacerbación de asma que puede resultar en hipoxemia, hipercarbia e insuficiencia respiratoria secundaria con manifestaciones clínicas agravadas que obligan al uso de ventilación mecánica con las complicaciones agregadas resultado del baro trauma.

Con relación a los factores ambientales de riesgo los resultados demuestran porcentajes elevados de exposición al humo de tabaco con su efecto inflamatorio ${ }^{14}$ y contacto permanente con perros y gatos con su reconocida sensibilización alérgica ${ }^{15}$, ambos desencadenantes de asma alérgica persistente, de difícil control y que eventualmente progresan a asma severa, como lo demuestra la literatura médica sobre el tema. Situaciones que son difíciles de eliminar por el trasfondo cultural alrededor de estas prácticas vivenciales que afectan la vida y el bienestar de los niños que padecen asma y cuyas complicaciones graves son prevenibles.

Estos resultados deben hacer reflexionar acerca de los factores que están incidiendo para que las complicaciones graves se den con tanta frecuencia. El enfoque debe dirigirse a la familia, a la atención primaria y al hospital. La población de estudio pertenece a estrato económico deprimido, donde no se considera prioritaria la salud, las familias están más preocupadas por la supervivencia/día, las enfermedades cuestan y la economía familiar no contempla este gasto. Las 


\section{Complicaciones graves en niños con asma}

Vol. 2, núm. 3., (2018)

Carmen Lazo Caicedo; Cesar Bravo Bermeo; Sonia Cedeño Loor; Eduardo Vera Muthre

viviendas son estrechas, mal iluminadas y las personas viven hacinadas, con animales al interior de las mismas. No tener conciencia de la gravedad de la enfermedad y de los factores de riesgo con los que conviven, estas condiciones y muchas otras contribuye a que el asma sea una enfermedad de alta prevalencia. Se suma las dificultades de acceso a los centros de atención primaria, por las distancias y los horarios, que las crisis de asma no contemplan.

Además, el inadecuado control y seguimiento de los pacientes, la carencia en las unidades de atención primaria de medicamentos y dispositivos que faciliten la adherencia al tratamiento o que impidan o retrasen las crisis, debido a los costos elevados. La falta de registro detallado, categorización de asma y educación preventiva a los familiares responsables del cuidado de los niños. El diagnóstico no oportuno y la identificación tardía de los pacientes con asma grave que presentan manifestaciones clínicas y parámetros de laboratorio indicativos de tomar medidas urgentes e instalar tratamientos y terapias ventilatorias que han demostrado eficacia en estos casos. La puesta al día de los hospitales tanto en recursos humanos y técnicos es una necesidad, también la educación continua de médicos tratantes y residentes en temas como el estatus asmático, condición grave, anteceden otras complicaciones como las registradas en este estudio. 


\section{Complicaciones graves en niños con asma}

Vol. 2, núm. 3., (2018)

Carmen Lazo Caicedo; Cesar Bravo Bermeo; Sonia Cedeño Loor; Eduardo Vera Muthre

\section{Bibliografía}

1. McFaden ER Jr, Warren EL. Observations on asthma mortality. Anns. Intern. Med. 1997; 127(2): 142-7.

2. National Asthma Education and Prevention Program. Guidelines for Diagnosis and Management of Asthma, Expert Panel Report 2. Bethesda: National Asthma Education and Prevention Program.1997.

3. Fitzpatrick AM, Teague WG, Meyers DA, Peters SP, Li X, Li H, Wenzek SE, Aujla, Castro M, Bacharier LB, et al. Heterogeneity of severe asthma in chilhood. Confirmation by cluster analysis of children in the national institutes of health/national heart,lung and blood institute severe asthma research program. J Allergy Clin Inunolol. 2011; 127(2):382-9e1-13

4. Moore BB, Weiss KB, Sullivan SA: Epidemiology and socioeconomic impact of asevere asthma. In Severe Asthma Pathogenesis and Clinical Management. Edited by Szefler SJ, Leun DYM. New York: Marcel Dekker Inc; 1996: 1-3.

5. Busse WW, Banks-Schlegel S, Wenzel SE: Pathophysiology of severe asthma. J Allergy Clin. Inmunol. 2000, 106(6): 1033-42.

6. Rampa S, Allareddy V, Asad R, Nalliah RP, Allareddy V, Rotta AT. Outcome of invasive mechanical ventilation in children and adolescents hospitalized due to status asthmaticus in the United States: A population based study. J. Ashtma. 2015; 52(4):42330 .

7. González García L, Rey C, Medina A, Mayordomo-Colunga J. Severe subcutaneous emphysema and pneumomediastinum secondary to noninvasive ventilation support in status asthmaticus. Indian J. Crit. Care. Med. 2016; 20(4):242-4.

8. Ricketti PA, Unkle DW, Lockey R, Cleri DJ, Ricketti AJ. Case study: Idiopathic hemothorax in a patient with status asthmaticus. J Asthma. 2016; 53 (7):770-3.

9. Tortajada-Girbes M., Moreno-Prat M, Ainsa-Laguna D, Mas S. Spontanoeus pneumomediastinun and subcutaneous emphysema as a complication of asthma in children: case report and literature review. Ther Ady Respir Dis. 2016; 10(5):402-9.

10. Miller AG, Breslin ME, Pineda LC, Fox JW. An Asthma Protocol Improved Adherence to Evidence-Based Guidelines for Pediatric Subjects with Status Asthmaticus in the Emergency Department. Respir Care. 2015; 60(12):1759-64. 


\section{Complicaciones graves en niños con asma}

Vol. 2, núm. 3., (2018)

Carmen Lazo Caicedo; Cesar Bravo Bermeo; Sonia Cedeño Loor; Eduardo Vera Muthre

11. De la Vega Pazitkova T., Pérez V., Bezos L. Factores de riesgo de asma bronquial en niños y su relacion con la severidad de manisfestaciones clínicas. Rev. Cubana. Med. Gen. Integr. 2010; 26(2):190-214.

12. Khadadah ME, Onadeko BO, Mustafa HT, Metwali KE. Clinical features and outcome of management of severe acute asthma (status asthmaticus) in the intensive care unit of a tertiary medical center. Singapore Med J 2000; 41(5):214-7.

13. Soubra S, Guntupalli K. Acute respiratory failure in asthma. Indian J Crit Car Med. 2005; 9(4):225-234

14. Combair SA, Gaston MB, Ricci KS, Hammel J, Dweik RA, Teague WG, Meyers D, Ampleford EJ, Bleeker ER, Busse WW, et al. Detrimental effects of environmental tobacco smoke in relation to asthma severity. PloS ONE 2011; 6(5):e 18574

15. Lockey R. The myth of hypoallergenic dogs (and cats). J Allergy Clin Inmunol 2012; 130(4):910-1 